\title{
ARTICLE OPEN \\ Adiabatic two-qubit gates in capacitively coupled quantum dot hybrid qubits
}

\author{
Adam Frees (D)', Sebastian Mehl ${ }^{2,3}$, John King Gamble ${ }^{4,5}$, Mark Friesen (D) ${ }^{1}$ and S. N. Coppersmith (iD)
}

The ability to tune qubits to flat points in their energy dispersions ("sweet spots") is an important tool for mitigating the effects of charge noise and dephasing in solid-state devices. However, the number of derivatives that must be simultaneously set to zero grows exponentially with the number of coupled qubits, making the task untenable for as few as two qubits. This is a particular problem for adiabatic gates, due to their slower speeds. Here, we propose an adiabatic two-qubit gate for quantum dot hybrid qubits, based on the tunable, electrostatic coupling between distinct charge configurations. We confirm the absence of a conventional sweet spot, but show that controlled-Z (CZ) gates can nonetheless be optimized to have fidelities of $\sim 99 \%$ for a typical level of quasistatic charge noise $\left(\sigma_{\varepsilon} \simeq 1 \mu \mathrm{eV}\right)$. We then develop the concept of a dynamical sweet spot (DSS), for which the time-averaged energy derivatives are set to zero, and identify a simple pulse sequence that achieves an approximate DSS for a CZ gate, with a $5 \times$ improvement in the fidelity. We observe that the results depend on the number of tunable parameters in the pulse sequence, and speculate that a more elaborate sequence could potentially attain a true DSS.

npj Quantum Information (2019)5:73; https://doi.org/10.1038/s41534-019-0190-7

\section{INTRODUCTION}

Since their original proposal, ${ }^{1}$ semiconductor quantum dot qubits have progressed greatly, demonstrating excellent qubit coherence and performance through the use of sweet spots ${ }^{2-12}$ and control of the spin degree of freedom. ${ }^{13-16}$ There has also been remarkable progress in systems with small numbers of donorbound electrons. ${ }^{17-25}$ Recently, two-qubit gates ${ }^{26,27}$ and algorithms ${ }^{28}$ have been realized using exchange-coupled single-spin qubits. Capacitive coupling has also been employed to entangle and perform two-qubit operations between singlet-triplet qubits, ${ }^{29,30}$ and has been proposed as the basis for two-qubit gates between resonant-exchange qubits ${ }^{31}$ and flip-flop qubits. ${ }^{23}$ In these experiments and proposals, the two-qubit gate times are typically measured in microseconds or hundreds of nanoseconds, which is much longer than typical single-qubit gate times. In contrast, the predicted two-qubit gate times for capacitively coupled quantum dot hybrid qubits ${ }^{32,33}$ (QDHQs) are comparable to single-qubit gates, which are of order $10 \mathrm{~ns} .^{10,34}$ However, the methods proposed in refs. ${ }^{32,33}$ rely on applying quickly varying electrical pulses, which can cause leakage from the qubit subspace. $^{34}$

In this paper, we study an adiabatic entangling protocol based on capacitive couplings between QDHQs. The gate is inspired by an early proposal for entangling singlet-triplet qubits. ${ }^{35}$ Although the necessary voltage changes are slow relative to the qubit frequencies, we show that high-fidelity adiabatic gates can be achieved in under $50 \mathrm{~ns}$, which is significantly faster than those in recent singlet-triplet experiments. ${ }^{29,30}$ While the pulse sequences used in adiabatic protocols are more resilient against pulse errors than nonadiabatic pulses and are less susceptible to leakage errors, a potential concern is that they could be more susceptible to charge noise due to slower speeds. It is, therefore, crucial to study the effect of charge noise on the gate fidelities.

We begin by considering the system of two capacitively coupled QDHQs, deriving the effective couplings between the two qubits, and describing how a slowly varying electrical pulse on the qubits can yield an entangling gate. Next, we optimize the pulse sequence for a two-qubit system to maximize the process fidelity of the resulting gate. We find that gate fidelities $>99 \%$ are feasible, assuming quasistatic charge noise with a standard deviation of $\sigma_{\varepsilon}=1 \mu \mathrm{eV}$, and that the infidelity scales roughly as $\sigma_{\varepsilon}^{2}$. Finally, we show that gate fidelities can be further improved to $\sim 99.9 \%$ by modifying pulse sequences to impose a "dynamical sweet spot," (DSS) a technique similar to dynamical decoupling. ${ }^{36}$

\section{RESULTS}

The QDHQ consists of three electrons shared between two quantum dots. The minimal Hilbert space of the qubit can be defined as the spin states $|\cdot S\rangle=|\downarrow\rangle|S\rangle,|\cdot T\rangle=\sqrt{\frac{1}{3}}|\downarrow\rangle\left|T_{0}\right\rangle-$ $\sqrt{\frac{2}{3}}|\uparrow\rangle\left|T_{-}\right\rangle$, and $|S \cdot\rangle=|S\rangle|\downarrow\rangle,{ }^{37,38}$ where $|\cdot S\rangle$ and $|\cdot T\rangle$ correspond to $(1,2)$ charge configurations (one electron in the left dot, two electrons in the right), $|S \cdot\rangle$ corresponds to a $(2,1)$ charge configuration, and the singlet state, $|S\rangle=1 / \sqrt{2}(|\downarrow \uparrow\rangle-|\uparrow \downarrow\rangle)$, and triplet states, $\left|T_{0}\right\rangle=1 / \sqrt{2}(|\downarrow \uparrow\rangle+|\uparrow \downarrow\rangle)$ and $\left|T_{-}\right\rangle=|\downarrow \downarrow\rangle$, refer to the dot with two electrons. In this basis, the single-qubit

\footnotetext{
'Department of Physics, University of Wisconsin-Madison, Madison, WI 53706, USA; ${ }^{2}$ JARA-Institute for Quantum Information, RWTH Aachen University, D-52056 Aachen, Germany; ${ }^{3}$ Peter Grünberg Institute (PGI-2), Forschungszentrum Jülich, D-52425 Jülich, Germany; ${ }^{4}$ Center for Computing Research, Sandia National Laboratories, Albuquerque, NM 87123, USA; ${ }^{5}$ Quantum Architectures and Computation Group, Microsoft Research, Redmond, WA 98052, USA and ${ }^{6}$ School of Physics, University of New South Wales, Sydney, NSW 2052, Australia

Correspondence: Adam Frees (adam.j.frees@gmail.com)
}

Received: 7 December 2018 Accepted: 6 August 2019

Published online: 02 September 2019 
a

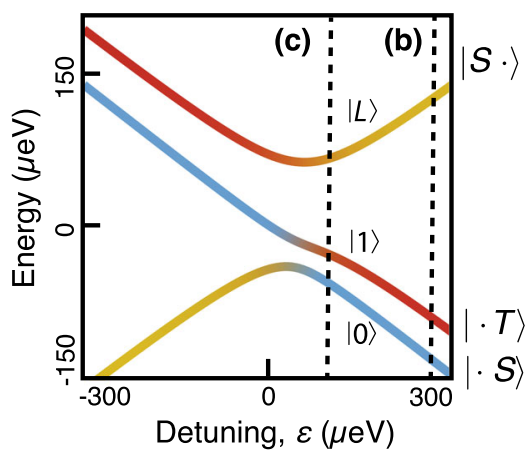

b
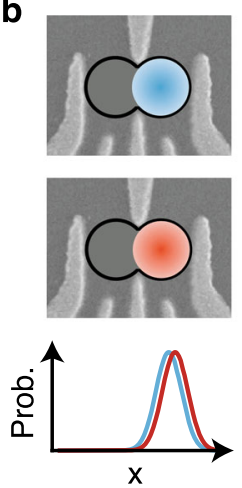

C

$|0\rangle$

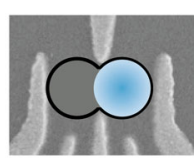

1)
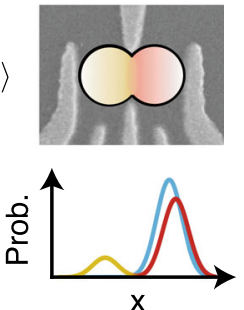

d

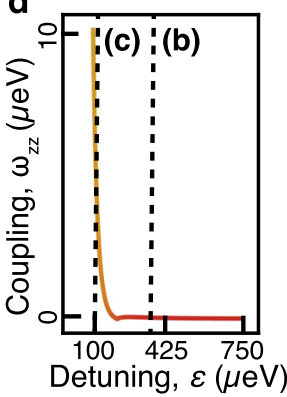

e

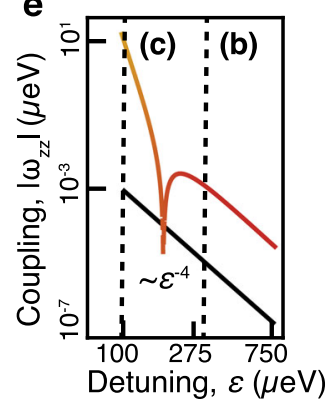

f

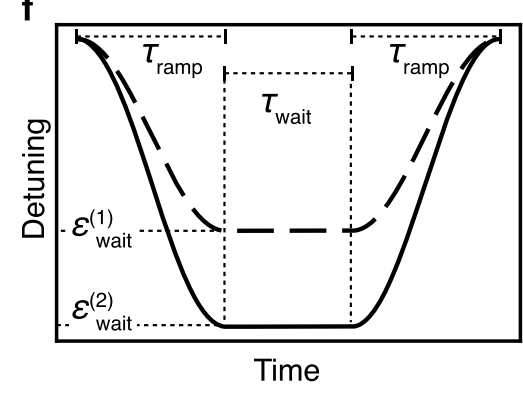

Fig. 1 Implementing a CZ gate between capacitively coupled QDHQs. a Energy dispersion of a single QDHQ as a function of detuning, as defined in Eq. (1), for typical experimental values given by [10] $\Delta_{1}=18.1 \mu \mathrm{eV}, \Delta_{2}=46.7 \mu \mathrm{eV}$, and $E_{\mathrm{ST}}=51.7 \mu \mathrm{eV}$. On the right-hand side of the plot, the logical states $|0\rangle$ and $|1\rangle$ converge to the basis states $|\cdot S\rangle$ (indicated in blue) and $|\cdot T\rangle$ (red), as defined in the main text, while the leakage state $|L\rangle$ converges to $|S \cdot\rangle$ (yellow). b, c Charge distributions of a third electron added to an underlying $(1,1)$ charge configuration at two different values of $\varepsilon$. $\mathbf{b}$ In the large- $\varepsilon$ regime (right-most dashed line in (a)), the qubit states have very similar charge distributions (same color coding as (a)). c For $\varepsilon$ near the charge transition (left-most dashed line in (a)), some charge moves from the right dot to the left dot, especially for state $|1\rangle$, setting up a dipole moment between states $|0\rangle$ and $|1\rangle$. d, e Effective two-qubit coupling versus $\varepsilon=\varepsilon^{(1)}=\varepsilon^{(2)}$, plotted on linear-linear (d) and log-log (e) scales. When $\varepsilon$ is large, the coupling is negligible and decreases as $\varepsilon^{-4}$ (see Eq. (S13)). When $\varepsilon$ is simultaneously lowered on both qubits, their dipole moments grow, and the effective coupling increases. $f$ Detuning pulse sequences for qubits 1 and 2 (dashed and solid lines, respectively; see Supplementary Section S3 ${ }^{42}$ for details)

Hamiltonian is

$\mathcal{H}_{1 q}=\left(\begin{array}{ccc}-\varepsilon / 2 & 0 & \Delta_{1} \\ 0 & -\varepsilon / 2+E_{\mathrm{ST}} & -\Delta_{2} \\ \Delta_{1} & -\Delta_{2} & \varepsilon / 2\end{array}\right)$,

where the detuning parameter, $\varepsilon$, corresponds to the energy separation between the quantum dots, $\Delta_{1}$ is the tunnel coupling between states $|\cdot S\rangle$ and $|S \cdot\rangle, \Delta_{2}$ is the tunnel coupling between states $|\cdot T\rangle$ and $|S \cdot\rangle$, and $E_{\mathrm{ST}}$ is the energy splitting between the singlet-like and triplet-like basis states, $|\cdot S\rangle$ and $|\cdot T\rangle$. A typical energy spectrum for $\mathcal{H}_{1 q}$ is plotted as a function of detuning in Fig. 1a. Here, the two lowest-energy eigenstates $|0\rangle$ and $|1\rangle$ form the qubit, while the remaining state $|L\rangle$ is regarded as a leakage state.

The basis states and charge configurations hybridize as a function of the detuning. The large detuning regime (right-most dashed line in Fig. 1a) is characterized by the asymptotic behavior $|0\rangle \simeq|\cdot S\rangle$ and $|1\rangle \simeq|\cdot T\rangle$, for which both states have the same charge configuration, as depicted in Fig. 1b. Here, the information is stored entirely in the spin degree of freedom and the qubit is well protected from charge noise; ${ }^{10}$ however, the single-qubit gate speeds can be slow. ${ }^{39,40}$ (Below, we show the same is true for twoqubit gates.) To perform efficient gates, we must therefore lower the detuning, bringing it near the anticrossing region (left-most dashed line in Fig. 1a). In this regime, $|\cdot S\rangle$ and $|\cdot T\rangle$ begin to hybridize with $|S \cdot\rangle$, which has a $(2,1)$ charge configuration, as depicted in Fig. 1c. Since the admixture of $(2,1)$ is different for $|0\rangle$ and $|1\rangle$, the qubit states acquire distinct dipole moments that can be used to mediate two-qubit dipole-dipole interactions, but which also couple to environmental charge noise. The goal of this work is to optimize the control parameters, to achieve high-fidelity two-qubit gates.

We can formalize the concept of a dipole moment by defining the operator $\hat{x}=\operatorname{diag}\{d / 2, d / 2,-d / 2\}$, describing the position of the third electron in the double dot, as depicted in Fig. 1b, c. Here, for simplicity, we assume that states $|\cdot S\rangle$ and $|\cdot T\rangle$ have identical charge configurations. The dimensionless dipole operator is, therefore, given by $\mathcal{P}=-\hat{x} / d=\operatorname{diag}\{-1 / 2,-1 / 2,1 / 2\}$, which is related to Eq. (1) through $\mathcal{P}=\partial \mathcal{H}_{1 q} / \partial \varepsilon$, where $\varepsilon$ plays the role of an electric field along the axis between the dots. The two-qubit Coulomb interaction can be expressed in terms of the dipole moments $\mathcal{P}^{(1)}$ and $\mathcal{P}^{(2)}$ of qubits 1 and 2 . We first note that the Coulomb interaction is classical, and therefore diagonal, when expressed in a charge-state basis. In analogy with charge qubits, the interaction can then be fully specified by $b^{(0)} \mathcal{I}^{(1)} \otimes \mathcal{I}^{(2)}+$ $b^{(1)} \mathcal{P}^{(1)} \otimes \mathcal{I}^{(2)}+b^{(2)} \mathcal{I}^{(1)} \otimes \mathcal{P}^{(2)}+b^{(3)} \mathcal{P}^{(1)} \otimes \mathcal{P}^{(2)}$. The first term in this expression is a uniform energy shift, which can be ignored. The second and third terms can be absorbed into the detuning parameters through the transformation $\varepsilon^{(i)} \rightarrow \varepsilon^{(i)}+b^{(i)}(i=1,2)$. Finally, defining $g$ as the change in Coulomb energy when one of the qubits flips its charge configuration, the two-qubit Hamiltonian becomes

$$
\mathcal{H}_{2 q}=\mathcal{H}_{1 q}^{(1)} \otimes I^{(2)}+I^{(1)} \otimes \mathcal{H}_{1 q}^{(2)}+g \mathcal{P}^{(1)} \otimes \mathcal{P}^{(2)}
$$

This form is generic and does not depend on qubit geometry. However, the value of $g$ depends on the geometry, and has been found to be of order $75 \mu \mathrm{eV}$ for a linear dot array. ${ }^{41}$ The full 9D basis set for Eq. (2) is given by $\{|\cdot S\rangle,|\cdot T\rangle,|S \cdot\rangle\}^{(1)} \otimes\{|\cdot S\rangle,|\cdot T\rangle,|S \cdot\rangle\}^{(2)}$, and 
the corresponding matrix representation for $\mathcal{H}_{2 q}$ is given in Supplementary Section S1. ${ }^{42}$

We first discuss qubit initialization and the implementation of single-qubit gates. In the large-detuning regime, the qubit logical states are energetically well separated from the leakage states, as shown in Supplementary Fig. S1, ${ }^{42}$ allowing leakage-free qubit initialization. To gain insight into gate operations, we perform a canonical transformation to decouple the logical states from the leakage states in the large-detuning limit. In addition, we evaluate this Hamiltonian in the adiabatic basis (the basis that diagonalizes $\mathcal{H}_{2 q}$ ), yielding the effective Hamiltonian

$$
\mathcal{H}_{2 q, \text { eff }} \simeq \frac{-\hbar \omega_{21}}{2} \sigma_{z}^{(1)} \otimes I^{(2)}+\frac{-\hbar \omega_{z 2}}{2} I^{(1)} \otimes \sigma_{z}^{(2)}+\frac{\hbar \omega_{z z}}{2} \sigma_{z}^{(1)} \otimes \sigma_{z}^{(2)},
$$

where the leading-order contributions to the single-qubit prefactors are of order $\hbar \omega_{z i}=E_{\mathrm{ST}}^{(i)}+\mathcal{O}\left[\Delta_{z}^{(i)^{2}} / \varepsilon^{(i)}\right]$, and the effective two-qubit coupling $\hbar \omega_{z z}$ is discussed below. (See Supplementary Section $S 1^{42}$ for details of the calculation.) Here, the sub- (or super)-script $i$ identifies the qubit, $\Delta_{z}^{(i)^{2}}$ is a quadratic function of the tunnel couplings, and the identity and Pauli matrices, $I^{(i)}$ and $\sigma_{z}^{(i)}$, act on the logical subspace. Although the detuning parameters provide some control over the qubit resonant frequencies, $\omega_{z 1}$ and $\omega_{z 2}, E_{\mathrm{ST}}$ typically varies significantly from dot to dot, resulting in well separated resonances. Single-qubit gates thus proceed by lowering one of the detunings (say, $\varepsilon^{(1)}$ ) from its high value to a regime where fast ac gates can be performed (e.g., the first dashed line in Fig. 1a). At this point, the dipole on qubit 1 is nonnegligible; however, we can operate it near a single-qubit sweet spot to minimize dephasing, as described in Supplementary Section $52 .{ }^{42}$ Since $\varepsilon^{(2)}$ remains at a large value, there is no danger of implementing either a singlequbit gate on qubit 2, or a two-qubit gate. Superimposing an ac drive on $\varepsilon^{(1)}$ at the resonant frequency of qubit 1 yields an additional term in Eq. (3) proportional to $\cos \left(\omega_{z 1} t\right) \sigma_{x}^{(1)} \otimes I^{(2)}$, which induces Rabi oscillations about the $\hat{\mathbf{x}}$-axis of the qubit; additional modulation of the phase in $\cos \left(\omega_{z 1} t+\phi\right)$ enables rotations about an arbitrary axis in the $x-y$ plane. To suppress the coupling of the dipole moment to external charge noise, we return $\varepsilon^{(1)}$ to its large value when the gate is finished.

Next, we consider two-qubit gate operations, which are performed adiabatically, and do not involve ac driving. The canonical transformation leading to Eq. (3) yields the leading order result at high detuning, $\hbar \omega_{z z}=\mathcal{O}\left[g \Delta^{4} / \varepsilon^{(1)^{2}} \varepsilon^{(2)^{2}}\right]$, where $\Delta^{4}$ is a quartic function of the tunnel couplings in both qubits. As anticipated, the adiabatic gate speed $\left|\omega_{z z}\right| / 2 \pi$ depends linearly on $g$, and requires $\varepsilon^{(1)}$ and $\varepsilon^{(2)}$ to be simultaneously reduced from their high values to initiate a two-qubit gate. The canonical transformation breaks down when $\varepsilon^{(1)}$ and $\varepsilon^{(2)}$ take their low values; however, under adiabatic operation, the projection onto the logical subspace, Eq. (3), is still meaningful. We can compute $\hbar \omega_{z z}$ at arbitrary detuning values by evaluating $\mathcal{H}_{2 q}$ in its adiabatic basis and projecting it onto the $4 \mathrm{D}$ logical subspace, $\mathcal{H}_{2 q} \rightarrow \mathcal{H}_{4 \mathrm{D}}$. We then identify $\hbar \omega_{z z}=\frac{1}{2} \operatorname{Tr}\left[\left(\sigma_{z}^{(1)} \otimes \sigma_{z}^{(2)}\right) \mathcal{H}_{4 \mathrm{D}}\right]=\frac{1}{2}\left(E_{00}-E_{01}-E_{10}+E_{11}\right)$, where $E_{i j}$ is the energy eigenvalue corresponding to the twoqubit logical state $|i j\rangle$. In Fig. 1d, e, we plot numerical results for $\hbar \omega_{z z}$ assuming typical qubit parameters and $\varepsilon \equiv \varepsilon^{(1)}=\varepsilon^{(2)}$. Here, we observe the predicted asymptotic behavior $\hbar \omega_{z z} \propto \varepsilon^{-4}$. We also note that $\hbar \omega_{z z}$ changes sign when $\varepsilon$ is of order $g$, in the lowdetuning regime where the canonical transformation breaks down.

A simple protocol for implementing adiabatic two-qubit gates is shown in Fig. 1f, and can be summarized as follows. We begin with the detuning parameters $\varepsilon^{(1)}$ and $\varepsilon^{(2)}$ set to "high" values of $500 \mu \mathrm{eV}$, and smoothly lower them to the "low" values $\varepsilon_{\text {wait }}^{(1)}$ and $\varepsilon_{\text {wait }}^{(2)}$ over a ramp time $\tau_{\text {ramp. }}$. The detunings are held constant at these values for a waiting period $\tau_{\text {wait }}$, and then smoothly returned
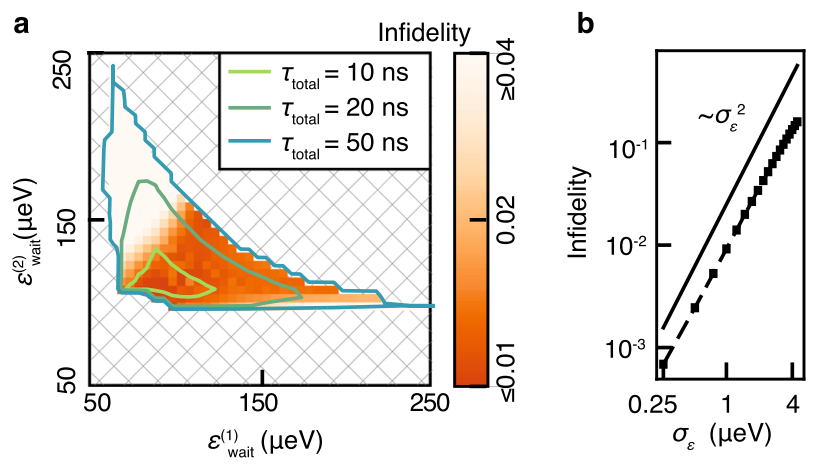

Fig. 2 Optimized infidelities of sub-50 ns adiabatic CZ gates. a Process infidelities obtained in the presence of quasistatic charge noise, with a standard deviation of $\sigma_{\varepsilon}=1 \mu \mathrm{eV}$; other Hamiltonian parameters are given in the main text. Following the optimization procedure described in "Methods", we obtain variable gate times, as indicated by the contours; however, we discard total gate times with $\tau_{\text {total }}=2 \tau_{\text {ramp }}+\tau_{\text {wait }}>50 \mathrm{~ns}$ (cross-hatched region) to ensure that the two-qubit gate is comparable in length to single-qubit gates. The highest fidelity pulse sequence is obtained at $\left(\varepsilon_{\text {wait }}^{(1)}, \varepsilon_{\text {wait }}^{(2)}\right)=(80,100) \mu \mathrm{eV}$. b Minimum CZ gate infidelities (black squares and dashed black line) plotted as a function of the standard deviation of the charge noise, $\sigma_{\varepsilon}$. The infidelity roughly falls off as $\sigma_{\varepsilon}^{2}$ (solid black line)

to $\varepsilon^{(1)}=\varepsilon^{(2)}=500 \mu \mathrm{eV}$ over the same ramp time $\tau_{\text {ramp. The }}$ parameters defining the pulse sequence are chosen to approximately yield a controlled-Z (CZ) gate operation. This protocol also produces incidental single-qubit $Z^{(1)}$ and $Z^{(2)}$ rotations, which can be eliminated, if necessary, by applying additional $Z^{(1)}$ and $Z^{(2)}$ gates. Explicit functional forms for $\varepsilon^{(1)}(t)$ and $\varepsilon^{(2)}(t)$ are given in Supplementary Section S3. ${ }^{42}$

We now compute the two-qubit gate fidelity for this sequence including both leakage and charge noise. While we do not explicitly consider the effects of relaxation error, in Supplementary Section $\mathrm{Sg}^{42}$ we provide a rough estimate of the expected $T_{1}$ times, finding them larger than the gate time. Leakage corresponds to the filling of quantum levels outside the logical subspace, and is primarily caused by nonadiabatic gate pulses. It is taken into account in our simulations by retaining the full 9D Hilbert space, comprising both logical and leakage states. In Methods section, we describe a method for computing the process fidelity of a CZ gate in the presence of charge noise. This procedure allows us to identify optimal values of $\tau_{\text {ramp }}$ and $\tau_{\text {wait }}$ consistent with fast pulse sequences, low leakage, and high fidelity. Figure 2 shows the results of such fidelity calculations, for a range of $\varepsilon_{\text {wait }}^{(1)}$ and $\varepsilon_{\text {wait }}^{(2)}$ values, assuming the typical quantum dot parameters $E_{\mathrm{ST}}^{(1)}=52 \mu \mathrm{eV}, E_{\mathrm{ST}}^{(2)}=47 \mu \mathrm{eV}, g=75 \mu \mathrm{eV}$, and $\sigma_{\varepsilon}=$ $1 \mu \mathrm{eV}$. Here, we choose $\Delta_{1}^{(i)}=0.64 E_{\mathrm{ST}}^{(i)}$ and $\Delta_{2}^{(i)}=0.58 E_{\mathrm{ST}}^{(i)}$, which suppresses the single-qubit dephasing, as discussed in Supplementary Section S2. ${ }^{42}$ We also omit pulse sequences with total gate times $\tau_{\text {total }}=2 \tau_{\text {ramp }}+\tau_{\text {wait }}>50$ ns (the cross-hatched regions in the plot), to ensure that entangling gates are performed on a timescale comparable to the QDHQ single-qubit gates. ${ }^{34}$ The fastest pulse sequence in the viable regime corresponds to $\varepsilon_{\text {wait }}^{(1)}=90 \mu \mathrm{eV}, \varepsilon_{\text {wait }}^{(2)}=110 \mu \mathrm{eV}, \tau_{\text {ramp }}=2.4 \mathrm{~ns}$, and $\tau_{\text {wait }}=2.8 \mathrm{~ns}$ $\left(\tau_{\text {total }}=7.6 \mathrm{~ns}\right)$, and exhibits an average process infidelity of $9.9 \times$ $10^{-3}$. The highest-fidelity sequence corresponds to $\varepsilon_{\text {wait }}^{(1)}=80 \mu \mathrm{eV}$, $\varepsilon_{\text {wait }}^{(2)}=100 \mu \mathrm{eV}, \tau_{\text {ramp }}=4.0 \mathrm{~ns}$, and $\tau_{\text {wait }}=8.0 \mathrm{~ns}\left(\tau_{\text {total }}=16.0 \mathrm{~ns}\right)$, with an average process infidelity of $9.2 \times 10^{-3}$. This optimized value depends on the standard deviation of the charge noise, $\sigma_{\varepsilon}$. In Fig. 2b, we plot the minimized CZ gate infidelity $\mathcal{I}$ as a function of $\sigma_{\varepsilon}$, revealing the scaling behavior $\mathcal{I} \propto \sigma_{\varepsilon}^{2}$. 
a

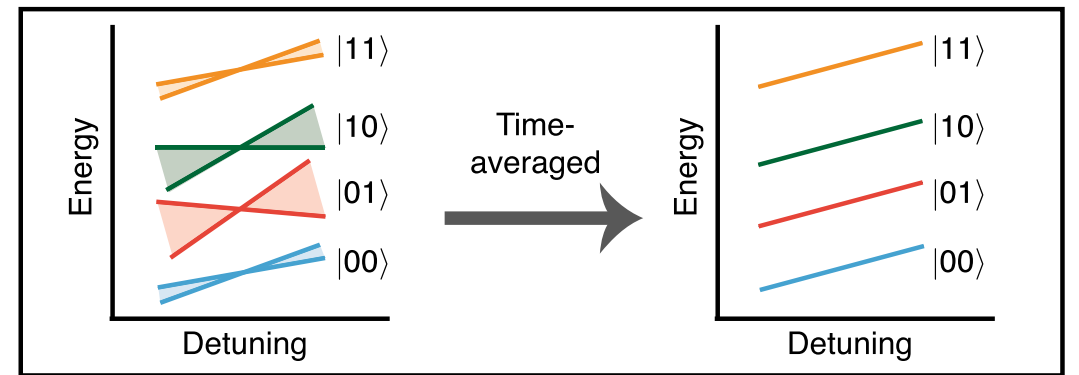

b

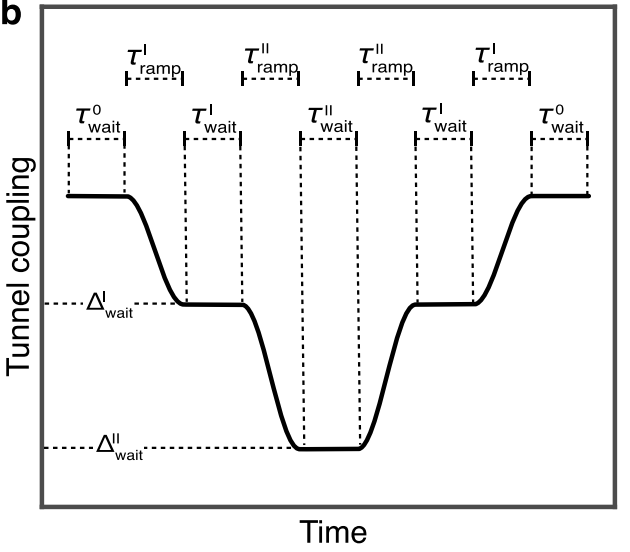

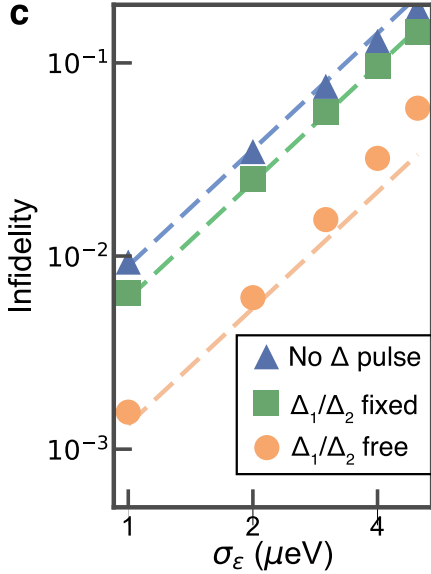

Fig. 3 Dynamical sweet spot (DSS) and a tunnel-coupling pulse sequence. a Two-qubit energy levels plotted schematically as a function of a single detuning variable. At any given time, it is difficult to arrange for all the energy dispersions to be parallel, as indicated on the left, leaving the qubits susceptible to dephasing. However, it may be possible to construct a pulse sequence for which the levels vary in time (shaded regions), such that their time-averaged dispersions are parallel, yielding a DSS that is more resilient to quasistatic fluctuations of the detuning. Here, we explore a DSS construction in which the detuning pulse sequence of Fig. $1 \mathrm{f}$ is augmented with the tunnel coupling pulse sequence defined in (b) and Supplemental Section S3. ${ }^{42}$ The latter is simple enough that it can be optimized using the method described in the main text, but complex enough that it provides a significant improvement in the CZ gate fidelity. c Infidelities calculated for three different pulse sequences as a function of the standard deviation of the quasistatic charge noise $\sigma_{\varepsilon}$. The markers correspond to full gate simulations averaged over a charge noise distribution, as described in "Methods". The dashed lines correspond to the much simpler infidelity estimate of Eq. (4). For the blue line and triangles, the tunnel couplings are held constant, as in Fig. 1. For the green line and squares, the tunnel couplings are pulsed as in (c) with the ratios $\Delta_{1}^{(i)} / \Delta_{2}^{(i)}$ held constant. For the orange line and circles, the tunnel coupling sequence is optimized with no constraints

The strong dependence of infidelity on $\sigma_{\varepsilon}$ indicates that dephasing, rather than leakage, is the main source of gate errors. In Supplementary Section S7, ${ }^{42}$ we explain the observed behavior by assuming that charge noise is quasistatic, obtaining

$\mathcal{I}_{\mathrm{cn}} \approx \frac{1}{4} \sigma_{\varepsilon}^{2} \sum_{i=1,2} \sum_{j=z 1, z 2, z z}\left(\int \frac{\partial \omega_{j}}{\partial \varepsilon^{(i)}} d t\right)^{2}$,

as expected in the absence of a sweet spot. ${ }^{6}$ To confirm the absence of a sweet spot, we perform an exhaustive search over the detuning $\left(\varepsilon^{(i)}\right)$, tunnel coupling $\left(\Delta_{j}^{(i)}\right)$, and Coulomb $(g)$ parameters in Eq. (2), finding that it is impossible to simultaneously set $\partial \omega_{j} / \partial \varepsilon^{(i)}=0$, for all $i$ and $j$, in the parameter range of interest. However, it is clear that this conventional, timeindependent definition of a sweet spot is overly restrictive for ensuring that $\mathcal{I}_{\mathrm{cn}} \approx 0$ in Eq. (4).

We now introduce the concept of a DSS in which the timeaveraged derivatives in Eq. (4) are made to vanish, as sketched in Fig. 3a. Through an exhaustive search (Fig. 2a), we have already demonstrated that no DSS exists for the simple pulse sequence of Fig. 1f. Moreover, because of the monotonic dependence of $\omega_{j}$ on $\varepsilon^{(i)}$ (for example, see Supplementary Fig. $\mathrm{S}^{42}$ ), it appears unlikely that a more elaborate detuning pulse sequence could provide significant improvements in the fidelity. We, therefore, augment the detuning sequence with a tunnel-coupling sequence, $\Delta_{j}^{(i)}(t)(i$, $j=1,2$ ). Our initial investigations suggest that a relatively large number of pulse parameters are needed to achieve significant improvements in the fidelity. We, therefore, consider the more elaborate pulse shape, shown in Fig. 3b, which incorporates seven parameters for each of four tunnel couplings.

Because of the large number of parameters in the combined detuning-tunnel-coupling sequence, we do not attempt an exhaustive search in this case. Instead, we maximize the CZ gate fidelity by performing a hundred separate Broyden-Fletcher-Goldfarb-Shanno ${ }^{43-46}$ searches using the method of ref. ${ }^{47}$, and adopting a basin-hopping protocol with randomized initial values to help escape any local minima. ${ }^{48}$ To simplify the calculation, we adopt the following hybrid infidelity functional: $\mathcal{I}_{\text {total }}=\mathcal{I}_{\mathrm{cn}}+\mathcal{I}_{\mathrm{nf}}+\mathcal{I}_{\text {na, }}$ which treats the charge noise (cn), noise-free (nf), and nonadiabatic (na) infidelity contributions separately. Calculating $\mathcal{I}_{\text {total }}$ is computationally efficient because all three contributions, including the charge noise term defined in Eq. (4), do not require taking an average over charge noise. The noise-free term describes the $C Z$ gate infidelity in the absence of charge noise, as described in "Methods". We find that minimizing just the $\mathcal{I}_{\mathrm{cn}}$ and $\mathcal{I}_{\text {nf }}$ terms (without $\mathcal{I}_{\text {na }}$ ) yields extremely short and fast pulse sequences that first populate then depopulate the leakage state. Since $\mathcal{I}_{\mathrm{cn}}$ was derived assuming an adiabatic pulse, these short and fast pulses are not guaranteed to have low process infidelity. Hence we also introduce the $\mathcal{I}_{\text {na }}$ term, as defined in Methods, to explicitly penalize nonadiabatic evolution.

We now obtain two different sets of solutions for the detuningtunnel-coupling pulse sequence. In the first, all the tunnel 
coupling parameters in Fig. $3 \mathrm{~b}$, as well as $\tau_{\text {ramp }}$ and $\tau_{\text {wait, }}$ are varied independently, under the constraint that the detuning and tunnel coupling sequences have the same length; this sequence contains 26 free parameters. The second case is similar, except that the ratio between the tunnel couplings in each double dot is assumed to be fixed throughout the sequence, with $\Delta_{1}^{(i)}(t) / \Delta_{2}^{(i)}(t)=1.1034$, as consistent with Supplementary Section $\mathrm{S}_{i}{ }^{42}$ this sequence contains 14 free parameters. In both cases, we set the detuning parameters to the values giving the fastest detuning-only pulse sequence in Fig. $2\left(\varepsilon_{\text {wait }}^{(1)}=90 \mu \mathrm{eV}\right.$ and $\left.\varepsilon_{\text {wait }}^{(2)}=110 \mu \mathrm{eV}\right)$, and we use $\tau_{\text {ramp }}=2.4 \mathrm{~ns}$ and $\tau_{\text {wait }}=2.8 \mathrm{~ns}$ as the starting points for our optimization procedure; initial values of the other parameters are chosen randomly, according to the basin-hopping protocol. The results of this procedure are presented in Supplementary Table $\mathrm{S} 1 .{ }^{42}$ Using these results, we recompute the infidelity as described in "Methods", performing an average over the charge noise.

Infidelity results using the tunnel coupling pulse sequence are plotted in Fig. $3 c$ as a function of charge noise, $\sigma_{\varepsilon}$. Here, we observe clear improvements compared to the detuning-only sequence, with the best results obtained for the sequence with the largest number of pulse parameters. Supplementary Section $\mathrm{S}^{42}$ suggests that this result can largely be attributed to the suppression of the time-averaged derivatives $\partial \omega_{j} / \partial \varepsilon^{(i)}$, as consistent with a DSS. For a true sweet spot, we would expect a powerlaw exponent in $\mathcal{I} \propto \sigma_{\varepsilon}^{a}$, with $a>2$. Although the large- $\sigma_{\varepsilon}$ data in Fig. $3 \mathrm{c}$ hint at such behavior, Supplementary Fig. $\mathrm{S}^{42}$ indicates that a full suppression of the time-averaged derivatives has not yet been achieved in the current pulse sequences.

\section{DISCUSSION}

We have proposed a scheme for entangling capacitively coupled quantum-dot hybrid qubits by applying adiabatic pulse sequences to detuning parameters. We have optimized the sequences in the presence of quasistatic charge noise and computed the resulting process fidelities for a CZ gate, obtaining fidelities approaching $99 \%$ for typical noise levels. Further improvements are obtained by simultaneously applying pulse sequences to the tunnel couplings. These results are explained by invoking the concept of a DSS, for which the splittings between the two-qubit energy levels are insensitive to fluctuations of the detuning parameters when averaged over the whole pulse sequence. Our analysis shows that a true DSS cannot be achieved using simple pulse sequences. However, fidelities $>99 \%$ are achieved when the pulse sequences include a large number of tunable parameters. As indicated by ref. ${ }^{49}$, these fidelities can be further improved by exploring a wider range of pulse shapes. Using the GRAPE algorithm, ${ }^{50}$ we have identified high-frequency pulses that significantly improve the expected performance under quasistatic charge noise. Despite the potential improvement in fidelity, the experimental bandwidth limitations make implementing some of these pulses impractical. We speculate that a bandwidth-limited version ${ }^{51,52}$ of the GRAPE algorithm $^{50}$ could be used to explore a much larger parameter space of adiabatic pulse sequences, possibly allowing us to identify a true DSS. The GRAPE algorithm also provides a means for exploring nonadiabatic pulse sequences. However, the simplicity and relatively high fidelity achieved with the sequences studied here, and the robustness of adiabatic gating methods, make the current proposal attractive for two-qubit gates.

\section{METHODS}

To study the time evolution resulting from the pulse sequences applied to capacitively coupled qubits, we numerically integrate the Hamiltonian in Eq. (2), for which the time-dependent control parameters $\tilde{\varepsilon}^{(1)}(t), \tilde{\varepsilon}^{(2)}(t)$, and $\vec{\Delta}(t)$ depend on the particular pulse sequence. Here, $\vec{\Delta}(t)$ refers to the set of four intra-qubit tunnel couplings, and we define $\tilde{\varepsilon}^{(i)}(t)=\varepsilon^{(i)}(t)+\delta \varepsilon^{(i)}$, where $\varepsilon^{(i)}(t)$ is the ideal, noise-free detuning sequence for qubit $i$, and the (quasi-static) noise term $\delta \varepsilon^{(i)}$ is assumed to remain constant for the duration of the sequence. The resulting unitary operator is given by

$$
U_{2 q}(t)=\exp \left[-i / \hbar \int_{0}^{t} \mathcal{H}_{2 q}\left(\varepsilon^{(1)}\left(t^{\prime}\right), \varepsilon^{(2)}\left(t^{\prime}\right), \vec{\Delta}\left(t^{\prime}\right)\right) d t^{\prime}\right] .
$$

In most cases, we take $t$ to be the final time in the pulse sequence, with one exception, described below.

We employ the following procedure to determine the detuning pulse parameters used in Fig. 2. (For additional details, see Section $\mathrm{S}^{42}$ of the Supplementary Materials). We first choose the fastest ramp time $\tau_{\text {ramp }}$ consistent with leakage errors $<0.1 \%$ in the absence of charge noise. We then compute $U_{2 q}$ as a function of $\tau_{\text {wait }}$ for a fixed level of quasi-static charge noise. (High-frequency noise can also affect the fidelity of slow QDHQ gates; ${ }^{53}$ however, we do not consider such processes here.) The process fidelity $\mathcal{F}$ is computed, comparing $U_{2 q}$ to a perfect $C Z$ gate, modulo single-qubit rotations, using the Choi-Jamiolkowski formalism, ${ }^{54}$ as described in Supplementary Section $55{ }^{42}$ We then average $\mathcal{F}$ over charge noise, using the method described below, and choose the value of $\tau_{\text {wait }}$ that maximizes $\langle\mathcal{F}\rangle$.

To optimize the detuning-tunnel-coupling pulse sequence used in Fig. 3, we choose pulse parameters that minimize the total infidelity function $\mathcal{I}_{\text {total }}=\mathcal{I}_{\mathrm{cn}}+\mathcal{I}_{\mathrm{nf}}+\mathcal{I}_{\mathrm{na}}$, as discussed in the main text. Here, the noise-free term $\mathcal{I}_{\mathrm{nf}}$ describes the $\mathrm{CZ}$ gate infidelity, computed using the Choi-Jamiolkowski formalism, as described above, in the absence of charge noise. In this work, we also introduce a penalty term to suppress nonadiabatic evolution, defined as $\mathcal{I}_{\text {na }}=\max _{t}\left[1-\frac{1}{4} \sum \mid\langle i j(t)| U_{2 q}\right.$ $\left.\left.(t)|i j(0)\rangle\right|^{2}\right]$, where the sum is taken over the logical basis states $(i, j)=(0$, 1 ), and the function $\max _{t}$ picks out the maximal occupation of leakage states, at any point in the pulse sequence. Note that the state $|i j(0)\rangle$ is an eigenstate of $H_{2 q}(t)$ at time $t=0$, while $|i j(t)\rangle$ is the corresponding eigenstate at time $t$. Under perfect adiabatic operation, the mapping $U_{2 q}(t) \mid$ $i j(0)\rangle \rightarrow|i j(t)\rangle$ is exact, yielding $\mathcal{I}_{\text {na }}=0$; however, for nonadiabatic operation, we obtain $\mathcal{I}_{\text {na }} g t ; 0$. In practice, we find that the exact form of $\mathcal{I}_{\text {na }}$ does not significantly affect our results.

To average the fidelity over charge noise, we assume that the noise terms $\delta \varepsilon^{(1)}$ and $\delta \varepsilon^{(2)}$ are uncorrelated and sample them independently at 17 values in the range between -25 and $+25 \mu \mathrm{eV}$, corresponding to 1089 unique pairs. We then linearly interpolate $\mathcal{F}$ over the results and calculate its average value, assuming a gaussian probability distribution with standard deviation $\sigma_{\varepsilon}$

$$
p\left(\delta \varepsilon^{(1)}, \delta \varepsilon^{(2)}\right)=\frac{1}{2 \pi \sigma_{\varepsilon}^{2}} \exp \left(-\frac{\delta \varepsilon^{(1) 2}+\delta \varepsilon^{(2) 2}}{2 \sigma_{\varepsilon}^{2}}\right) .
$$

\section{DATA AVAILABILITY}

The main data supporting the finding of this study are available within the article and its Supplementary Information files. Additional data can be provided upon request.

\section{ACKNOWLEDGEMENTS}

We thank D. Bradley, C. King, and R. Blume-Kohout for useful discussions. We also thank the HEP, Condor, and CHTC groups at University of Wisconsin-Madison for computational support. This work was supported in part by ARO (W911NF-12-1-0607 and W911NF-17-1-0274) and the Vannevar Bush Faculty Fellowship program sponsored by the Basic Research Office of the Assistant Secretary of Defense for Research and Engineering and funded by the Office of Naval Research through Grant no. N00014-15-1-0029. The views and conclusions contained in this document are those of the authors and should not be interpreted as representing the official policies, either expressed or implied, of the Army Research Office (ARO), or the U.S. Government. The U.S. Government is authorized to reproduce and distribute reprints for Government purposes notwithstanding any copyright notation herein. This paper describes objective technical results and analysis. Any subjective views or opinions that might be expressed in the paper do not necessarily represent the views of the U.S. Department of Energy or the United States Government. Sandia National Laboratories is a multimission laboratory managed and operated by National Technology \& Engineering Solutions of Sandia, LLC, a wholly owned subsidiary of Honeywell International Inc., for the U.S. Department of Energy ${ }^{\mathrm{TM}}$ National Nuclear Security Administration under contract DE-NA0003525. The authors gratefully acknowledge support from the Sandia National Laboratories Truman Fellowship Program, which is funded by the Laboratory Directed Research and Development (LDRD) Program. 


\section{AUTHOR CONTRIBUTIONS}

All authors contributed to the idea of using adiabatic gate sequences to entangle capacitively coupled QDHQs. A.F. performed the Schrieffer-Wolff transformation. A.F. and J.K.G. performed the numerical simulation, and analyzed the data with M.F. and S.N.C. A.F., J.K.G., M.F., and S.N.C. wrote the paper and prepared the figures, with input from all the authors.

\section{ADDITIONAL INFORMATION}

Supplementary information accompanies the paper on the npj Quantum Information website (https://doi.org/10.1038/s41534-019-0190-7).

Competing interests: The authors declare no competing interests.

Publisher's note: Springer Nature remains neutral with regard to jurisdictional claims in published maps and institutional affiliations.

\section{REFERENCES}

1. Loss, D. \& DiVincenzo, D. P. Quantum computation with quantum dots. Phys. Rev. A 57, 120-126 (1998).

2. Petersson, K. D., Petta, J. R., Lu, H. \& Gossard, A. C. Quantum coherence in a oneelectron semiconductor charge qubit. Phys. Rev. Lett. 105, 246804 (2010).

3. Dial, O. E. et al. Charge noise spectroscopy using coherent exchange oscillations in a singlet-triplet qubit. Phys. Rev. Lett. 110, 146804 (2013).

4. Kim, D. et al. Quantum control and process tomography of a semiconductor quantum dot hybrid qubit. Nature 511, 70-74 (2014).

5. Kim, D. et al. Microwave-driven coherent operation of a semiconductor quantum dot charge qubit. Nat. Nano 10, 243-247 (2015).

6. Cao, G. et al. Tunable hybrid qubit in a GaAs double quantum dot. Phys. Rev. Lett. 116, 086801 (2016).

7. Reed, M. D. et al. Reduced sensitivity to charge noise in semiconductor spin qubits via symmetric operation. Phys. Rev. Lett. 116, 110402 (2016).

8. Martins, F. et al. Noise suppression using symmetric exchange gates in spin qubits. Phys. Rev. Lett. 116, 116801 (2016).

9. Schoenfield, J. S., Freeman, B. M. \& Jiang, H. Coherent manipulation of valley states at multiple charge configurations of a silicon quantum dot device. Nat. Commun. 8, 64 (2017).

10. Thorgrimsson, B. et al. Extending the coherence of a quantum dot hybrid qubit. npj Quantum Inf. 3, 32 (2017).

11. Mi, X. et al. A coherent spin-photon interface in silicon. Nature 555, 599 (2018).

12. Samkharadze, N. et al. Strong spin-photon coupling in silicon. Science 359, 1123-1127 (2018).

13. Kawakami, E. et al. Gate fidelity and coherence of an electron spin in an Si/SiGe quantum dot with micromagnet. Proc. Natl Acad. Sci. 113, 11738-11743 (2016).

14. Yoneda, J. et al. A quantum-dot spin qubit with coherence limited by charge noise and fidelity higher than 99.9\%. Nat. Nanotechnol. 13, 102-106 (2018).

15. Crippa, A. et al. Electrical spin driving by $g$-matrix modulation in spin-orbit qubits. Phys. Rev. Lett. 120, 137702 (2018).

16. Jock, R. M. et al. A silicon metal-oxide-semiconductor electron spin-orbit qubit. Nat. Commun 9, 1768, https://doi.org/10.1038/s41467-018-04200-0 (2018).

17. Pla, J. J. et al. A single-atom electron spin qubit in silicon. Nature 489, 541-545 (2012).

18. Fuechsle, M. et al. A single-atom transistor. Nat. Nano 7, 242-246 (2012).

19. $\mathrm{Pla}, \mathrm{J}$. J. et al. High-fidelity readout and control of a nuclear spin qubit in silicon. Nature 496, 334-338 (2013).

20. Watson, T. F., Weber, B., House, M. G., Büch, H. \& Simmons, M. Y. High-fidelity rapid initialization and read-out of an electron spin via the single donor $D^{-}$ charge state. Phys. Rev. Lett. 115, 166806 (2015).

21. Laucht, A. et al. A dressed spin qubit in silicon. Nat. Nanotechnol. 12, 61 (2016).

22. Watson, T. F. et al. Atomically engineered electron spin lifetimes of $30 \mathrm{~s}$ in silico. Sci. Adv. 3, e1602811 (2017).

23. Tosi, G. et al. Silicon quantum processor with robust long-distance qubit couplings. Nat. Commun. 8, 450 (2017).

24. Harvey-Collard, P. et al. Coherent coupling between a quantum dot and a donor in silicon. Nat. Commun. 8, 1029 (2017).

25. Broome, M. A. et al. Two-electron spin correlations in precision placed donors in silicon. Nat. Commun. 9, 980 (2018).

26. Veldhorst, M. et al. A two-qubit logic gate in silicon. Nature 526, 410-414 (2015).

27. Zajac, D. M. et al. Resonantly driven CNOT gate for electron spins. Science $\mathbf{3 5 9}$, 439-442 (2018).

28. Watson, T. F. et al. A programmable two-qubit quantum processor in silicon. Nature 555, 633 (2018).
29. Shulman, M. D. et al. Demonstration of entanglement of electrostatically coupled singlet-triplet qubits. Science 336, 202-205 (2012).

30. Nichol, J. M. et al. High-fidelity entangling gate for double-quantum-dot spin qubits. npj Quantum Inf. 3, 3 (2017).

31. Taylor, J. M., Srinivasa, V. \& Medford, J. Electrically protected resonant exchange qubits in triple quantum dots. Phys. Rev. Lett. 111, 050502 (2013).

32. Mehl, S. Quantum computation with three-electron double quantum dots at an optimal operation point. Preprint at https://arxiv.org/abs/1507.03425 (2015).

33. Ferraro, E., Fanciulli, M. \& De Michielis, M. Controlled-NOT gate sequences for mixed spin qubit architectures in a noisy environment. Quantum Inf. Process. 16 (2017).

34. Kim, D. et al. High-fidelity resonant gating of a silicon-based quantum dot hybrid qubit. Npj Quantum Inf. 1, 15004 (2015).

35. Taylor, J. M. et al. Fault-tolerant architecture for quantum computation using electrically controlled semiconductor spins. Nat. Phys. 1, 177 EP- (2005).

36. Viola, L., Knill, E. \& Lloyd, S. Dynamical decoupling of open quantum systems. Phys. Rev. Lett. 82, 2417-2421 (1999).

37. Shi, Z. et al. Fast hybrid silicon double-quantum-dot qubit. Phys. Rev. Lett. 108, 140503 (2012).

38. Koh, T. S., Gamble, J. K., Friesen, M., Eriksson, M. A. \& Coppersmith, S. N. Pulsegated quantum-dot hybrid qubit. Phys. Rev. Lett. 109, 250503 (2012).

39. Wong, C. H. High-fidelity ac gate operations of a three-electron double quantum dot qubit. Phys. Rev. B 93, 035409 (2016).

40. Yang, Y.-C., Coppersmith, S. N. \& Friesen, M. Achieving high-fidelity single-qubit gates in a strongly driven silicon-quantum-dot hybrid qubit. Phys. Rev. A 95, 062321 (2017).

41. Ward, D. R. et al. State-conditional coherent charge qubit oscillations in a Si/SiGe quadruple quantum dot. Npj Quantum Inf. 2, 16032 (2016).

42. Frees, A. et al. Supplemental information for "Adiabatic two-qubit gates in capacitively coupled quantum dot hybrid qubits".

43. Broyden, C. G. The convergence of a class of double-rank minimization algorithms 1. General considerations. IMA J. Appl. Math. 6, 76-90 (1970).

44. Fletcher, R. A new approach to variable metric algorithms. Comput. J. 13, 317-322 (1970).

45. Goldfarb, D. A family of variable-metric methods derived by variational means. Math. Comput. 24, 23-26 (1970).

46. Shanno, D. F. Conditioning of quasi-vnewton methods for function minimization. Math. Comput. 24, 647-656 (1970).

47. Jones, E. et al. SciPy: Open source scientific tools for Python (2001) http://www. scipy.org/. Online; accessed 18 May 2018.

48. Leary, R. H. Global optimization on funneling landscapes. J. Glob. Optim. 18, 367383 (2000).

49. Setser, A. A., \& Kestner, J. P. Rapid adiabatic gating for capacitively coupled quantum dot hybrid qubits without barrier control. Phys. Rev. B 99, 195403, https://doi.org/10.1103/PhysRevB.99.195403 (2019).

50. Khaneja, N., Reiss, T., Kehlet, C., Schulte-Herbrüggen, T. \& Glaser, S. J. Optimal control of coupled spin dynamics: design of NMR pulse sequences by gradient ascent algorithms. J. Magn. Reson. 172, 296-305 (2005).

51. Lucarelli, D. Quantum optimal control via gradient ascent in function space and the time-bandwidth quantum speed limit. Phys. Rev. A 97, 062346 (2018).

52. Frey, V. M. et al. Application of optimal band-limited control protocols to quantum noise sensing. Nat. Commun. 8, 2189 (2017).

53. Gamble, J. K., Friesen, M., Coppersmith, S. N. \& Hu, X. Two-electron dephasing in single Si and GaAs quantum dots. Phys. Rev. B 86, 035302 (2012).

54. Gilchrist, A., Langford, N. K. \& Nielsen, M. A. Distance measures to compare real and ideal quantum processes. Phys. Rev. A 71, 062-310 (2005).

Open Access This article is licensed under a Creative Commons Attribution 4.0 International License, which permits use, sharing, adaptation, distribution and reproduction in any medium or format, as long as you give appropriate credit to the original author(s) and the source, provide a link to the Creative Commons license, and indicate if changes were made. The images or other third party material in this article are included in the article's Creative Commons license, unless indicated otherwise in a credit line to the material. If material is not included in the article's Creative Commons license and your intended use is not permitted by statutory regulation or exceeds the permitted use, you will need to obtain permission directly from the copyright holder. To view a copy of this license, visit http://creativecommons. org/licenses/by/4.0/.

(c) The Author(s) 2019 